\title{
A Method Combining Order Tracking and Fuzzy C-Means for Diesel Engine Fault Detection and Isolation
}

\author{
Ruili Zeng, ${ }^{1,2}$ Lingling Zhang, ${ }^{1}$ Yunkui Xiao, ${ }^{1}$ Jianmin Mei, \\ Bin Zhou, ${ }^{1}$ Huimin Zhao, ${ }^{1}$ and Jide Jia ${ }^{1}$ \\ ${ }^{1}$ Department of Automobile Engineering, Military Transportation University, Tianjin 300161, China \\ ${ }^{2}$ School of Precision Instrument and Opto-Electronics Engineering, Tianjin University, Tianjin 300072, China \\ Correspondence should be addressed to Ruili Zeng; zruili@163.com
}

Received 29 September 2014; Accepted 15 February 2015

Academic Editor: Changjun Zheng

Copyright (C) 2015 Ruili Zeng et al. This is an open access article distributed under the Creative Commons Attribution License, which permits unrestricted use, distribution, and reproduction in any medium, provided the original work is properly cited.

\begin{abstract}
Diesel engine works under variable speed conditions; fault symptoms are clearer in the angular/order domains than in the common time/frequency ones. In this paper, firstly, the acceleration signal of diesel engine is resampled by order tracking, in which the rotating speed is computed in every working cycle, and the order tracking spectrum is created in each interval's speed; then different order band accumulated energy is computed as feature vector. After standardizing these features, the fuzzy c-means (FCM) is introduced to use them as input vector; the optimized classified matrix and clustering centers can be obtained using FCM iteration method; then the fault can be detected by calculating the approach degree between the unknown samples and the known ones. To validate the method, some experiments have been performed; the results show that the signal can be reconstructed, and the features of order band accumulated energy can reflect the information of different wear conditions in crank-shaft bearing; then the fault can be detected accurately. The method of nonentire work cycle is also introduced as a comparison with our method; the result shows our method has more accuracy classification.
\end{abstract}

\section{Introduction}

The vibration signal in diesel engine includes some useful information, and the vibration signal in the process of diesel engine's acceleration or deceleration is captured usually to detect the faults because the mechanical fault symptoms are more apparent under the variable speed working conditions of diesel engine [1]. However, there is not an obvious boundary in mechanical faults caused by different degree of wear, and there are some cross terms in different fault samples; the fault classification is very vague; hence, this means that mechanical fault detection of diesel engine is very difficult. Actually, there are some methods reported on the analysis of the nonstationary signals [2-5], for example, short-time Fourier transform, wavelet transform, Hilbert-Huang transform, and cyclostationarity. These methods are influenced by noise, especially for the early weak fault, and some methods may cause frequency aliasing.
Based on the above discussion, we develop a combined method for diesel engine fault detection and isolation, which is based on the order tracking and FCM. Order tracking focuses mainly on the signal component associated with the speed, which transforms the nonstationary signals in the time domain to stationary signals in order domain for calculation of the power spectrum. Hence, the vibration signal under the variable speed working conditions can be analyzed by order tracking, and the features can be extracted easily [6-8]. FCM is an unsupervised machine learning technique, which can objectively classify the categories with fuzzy characteristics through uncertainty description of the sample class and become an important tool in pattern recognition, image processing, fuzzy control, and other areas $[9,10]$. Because the fault classification is very vague in mechanical fault of diesel engine, we can use this method to solve the problem of pattern recognition in engine fault detection and isolation. 


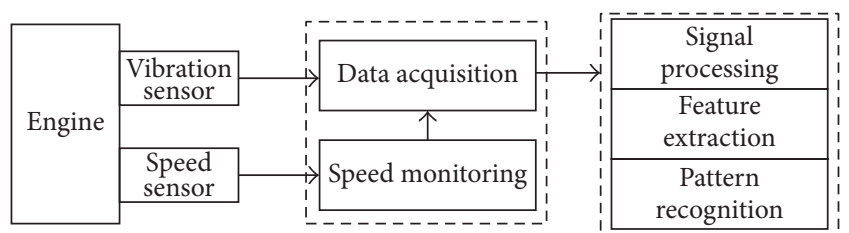

FIGURE 1: The framework of measurement system.

In order to detect engine fault, we designed a measurement system which is shown in Figure 1. We used vibration sensor to capture the vibration signal from diesel engine when it was running and speed sensor to monitor rotating speed of the engine simultaneously; then computer could get the vibration and speed signals including stationary and nonstationary signals under the variable speed working conditions of diesel engine. According to the method of order tracking, the signals were analyzed and the features were extracted. Then, we used FCM to recognize the engine's conditions.

In this paper, an analysis method about combining order tracking with FCM was presented, in which the order tracking spectrum in the entire work cycle of engine is calculated, and the different band energy of order tracking spectrum is computed as feature vector; then the cluster centers of samples are obtained by FCM method. Finally, the Hamming approach degrees of the cluster centers are calculated between test samples and known samples to classify the fault samples. Hence, we use this method to detect crankshaft bearings fault in diesel engine, and some experiments are performed to validate the analysis results.

\section{A Method Combining Order Tracking and Fuzzy C-Means}

\subsection{Order Tracking}

2.1.1. The Main Idea of Order Tracking. According to the theory of order tracking, machines are assumed as uniform variable rotation, and then rotation angle is a quadratic function of time, as follows [9]:

$$
\theta(t)=b_{0}+b_{1} t+b_{2} t^{2}
$$

where $\theta(t)$ is the rotation angle of crankshaft, $b_{0}, b_{1}$, and $b_{2}$ are the polynomial coefficients, respectively, and $t$ is time.

In order to find the polynomial coefficients of (1), we rewrite (1) as follows:

$$
\left[\begin{array}{l}
\theta_{1} \\
\theta_{2} \\
\theta_{3}
\end{array}\right]=\left[\begin{array}{lll}
1 & t_{1} & t_{1}^{2} \\
1 & t_{2} & t_{2}^{2} \\
1 & t_{3} & t_{3}^{2}
\end{array}\right]\left[\begin{array}{l}
b_{0} \\
b_{1} \\
b_{2}
\end{array}\right],
$$

where $t_{1}, t_{2}$, and $t_{3}$ are the times of three successive speed pulse signals, respectively, and $\theta_{1}, \theta_{2}$, and $\theta_{3}$ are the angles of the speed pulse signal intervals, respectively.
Based on (2), the corresponding angle change time can be solved as follows:

$$
t_{k}=\frac{1}{2 b_{2}}\left(\sqrt{b_{1}^{2}+4 b_{2}\left(\theta_{k}-b_{0}\right)}-b_{1}\right),
$$

where $t_{k}$ is the time corresponding to the position of angle and $\theta_{k}$ is the position of angle.

According to (3), the time of the same angular resampling sampling interval can be obtained, and the signal can be reconstructed after resampling by interpolation method.

In the method of speed signal processing based on a quadratic polynomial, there will be calculation error when some speed pulses are lost or there is additional pulse interference. In this paper, we process the speed pulse signal during acceleration; the speed signals are arranged with sort ascending then retain the first speed signal after removing other points among the same speed signals. After these, the speed signal can be revised.

2.1.2. Order Tracking Spectrum in the Entire Work Cycle. The vibration characteristics of the engine during acceleration will be obvious by order tracking analysis. Usually, the order tracking spectrum from a fixed speed is insignificant; however, if the engine were accelerated from idle speed to high speed, the order tracking spectrum in different speed sections with certain interval could be calculated; then, we can get the order tracking spectrum in the process of acceleration. For rotating machinery, the speed is generally divided into some intervals.

Actually, the engine can complete a work cycle after crankshaft rotating 2 laps due to the engine being special rotating reciprocating machinery; then a working cycle of engine is used as an interval. Hence, the order tracking spectrum in these intervals is calculated and put together and the order tracking spectrum of the entire working cycle will be obtained. These show the character of the signal with variable speed but also make the order tracking spectrum in different conditions more comparable.

2.1.3. Speed Signal Processing. Speed signal processing is a very important part of the order tracking analysis. In order to estimate the instantaneous frequency of order tracking, an accurate reference shaft speed curve is needed. Usually, this can be calculated by pulse of speed sensor. If the speed signals from the measurement channel were not good and then signal from other channels was better, we cannot get good order tracking results. Therefore, the higher accurate speed signal means the more accurate order tracking.

However, the speed signal obtained by the actual measurement will miss the pulses or extra pulses due to noise and vibration; hence, the obtained speed curve has a great error as shown in Figure 2(a); it is obvious that there are fluctuations around $2200 \mathrm{rpm}$ during acceleration; this will result in no solution in (2). Hence, it is necessary to process the speed signal. The speed signal will be distributed from small to large after collecting the signal. Then, the same rotational speed 


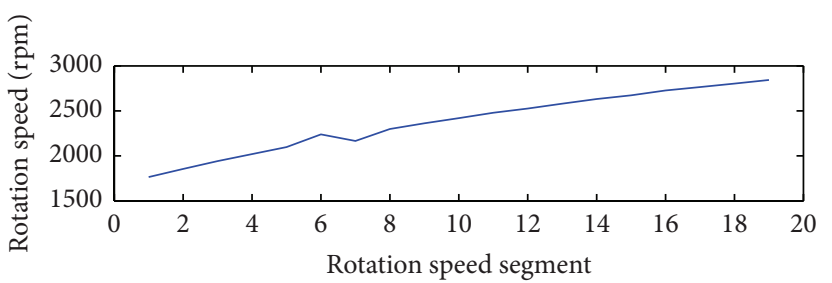

(a) The original rotation speed

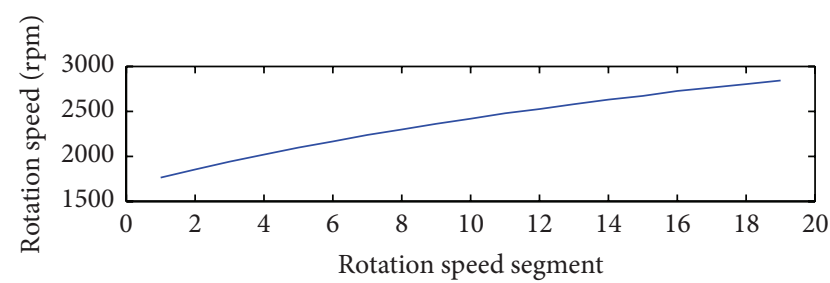

(b) The rotation speed after removing error signal

FIGURE 2: Speed curve of acceleration process.

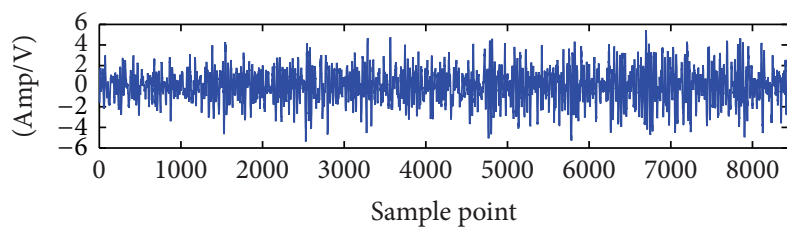

(a) The original vibration signal

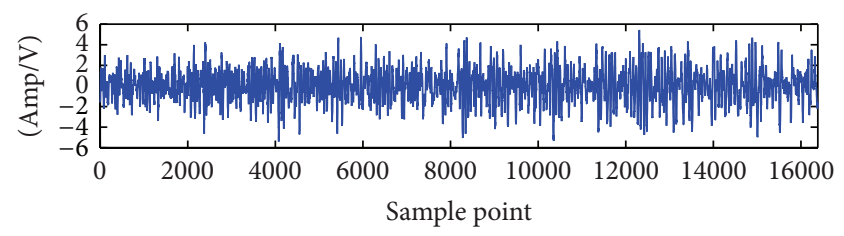

(b) The vibration signal after resampling

FIGURE 3: Vibration signal of acceleration process.

is removed and only the first one is retained. Because the rotation speed signal and vibration signal are synchronous captured, the vibration signal will be also removed when removing the error rotational speed, and the new rotation speed and vibration signal remain synchronized. After this process, the error from missing or additional pulses can be eliminated. Figure 2(b) is the speed curve after modification, where the rotation speed segment is the number of working cycles computed through rotation speed sensor's signal. It should be noted that the vibration signal related with the speed can also perform the appropriate sorting and removing. Hence, the angle of the sampling time can be gotten by solving (2). The resampled signal can be obtained by cubic spline interpolation method. The vibration signal of acceleration process $(1500 \mathrm{rpm}-2900 \mathrm{rpm})$ in time domain is shown in Figure 3(a), and the resampled signal is shown in Figure 3(b). According to Figure 3(b), the sample point of the signal is increased after resampling.

2.1.4. Features Extraction in Order Tracking Spectrum. In the order tracking spectrum, different energy band of order band can reflect the engine's conditions. In this paper, we use the accumulated energy of order band to reflect the engine's conditions. The signals of known samples are resampled, and some order bands are divided at regular interval in the whole order domain. Then, the accumulated energies of these order bands are calculated. We use Teager energy operator to calculate the accumulated energy, as follows:

$$
E(j)=\sum_{i=j}^{j+l}\left(S_{(i)}^{2}-S_{(i-1)} S_{(i+1)}\right),
$$

where $E(j)$ is the accumulated energy of the order band. $j$ is the number of the order band, $l$ is the interval, and $S_{(i)}$ is the signal after resampling. After these, we find out the maximum of $E(j)$, as follows:

$$
F=\max \{E(1), E(2), \ldots, E(j), \ldots, E(k)\} .
$$

Then, the interval $l$ is changed, and we can find the $F$ which is the most sensitive to known samples with different wear conditions. Hence, we divide the order tracking spectrum into different order band of the acceleration process and then use the accumulated energy of order band as features.

\subsection{Fuzzy C-Means and Selection Nearness Principle}

2.2.1. FCM Algorithm. FCM clustering is an unsupervised dynamic method. The sample space $x=\left\{x_{1}, x_{2}, \ldots, x_{n}\right\}$ is divided into $c$ classes $(2 \leq c \leq n)$ in this method. Arbitrary sample point $x_{i}$ is not strictly classified as a class. The membership belongs to the $j$ th $(1 \leq j \leq c)$ class of the arbitrary sample which is defined as $u_{i j}\left(0 \leq u_{i j} \leq 1\right)$. If a certain type of sample is considered to be a fuzzy subset of the sample set of $x$, the corresponding membership matrix will be a fuzzy membership matrix, which is denoted as $U=\left\{u_{i j}\right\}$. Then $U$ has the following characteristics [10-12]:

$$
\begin{gathered}
0 \leq u_{i j} \leq 1, \\
\sum_{j=1}^{c} u_{i j}=1, \\
0<\sum_{i=1}^{n} u_{i j}<n .
\end{gathered}
$$

FCM algorithm is to make the objective function $J_{\mathrm{fcm}}$ minimization in the constraints of (6). Therefore,

$$
J_{\mathrm{fcm}}(U, C)=\sum_{i=1}^{n} \sum_{j=1}^{c} u_{i j}^{m} d_{i j}\left(x_{i}, c_{j}\right),
$$


where $m$ is the fuzzy weighted index and $m>1 . c_{j}$ is the center of the $j$ th class in $c$ classes. $d_{i j}^{2}\left(x_{i}, c_{j}\right)=\left\|x_{i}-c_{j}\right\|$ is the Euclidean distance from sample point $\left(x_{i}\right)$ to the center of class $\left(c_{i}\right)$.

FCM algorithm uses an iterative approach to minimize the objective function, and the main idea is as follows.

Step 1. The number of classes is given as $c$, and the fuzzy weighted index is $m$. The stop threshold of iteration is set as $\varepsilon$, the iteration number is $k=0$, and the maximum iteration number is $k_{\max }$. Then, membership matrix $U^{(k)}$ is initialized by constraints.

Step 2. The cluster center is calculated by the membership matrix:

$$
c_{j}=\frac{\sum_{i=1}^{n} u_{i j}^{m} x_{i}}{\sum_{i=1}^{n} u_{i j}} .
$$

Step 3. The membership matrix $U^{(k+1)}$ is updated by the cluster center $c_{j}$ :

$$
u_{i j}^{(k+1)}=\left[\sum_{l=1}^{c}\left(\frac{d_{i j}^{(k+1)}}{d_{i l}^{(k+1)}}\right)^{2 /(m-1)}\right]^{-1}, \quad l=1,2, \ldots, c .
$$

Step 4. The convergence criterion is given as $\varepsilon>0$. If $\left\|U^{(k+1)}-U^{(k)}\right\| \leq \varepsilon$, then the iteration will be stopped; otherwise, $k=k+1$, and the calculation will go to Step 2 .

Step 5. An optimal fuzzy classification $U=\left\{u_{i j}\right\}$ about sample $x$ is gotten, and the class center $C=\left\{c_{j}\right\}$ is obtained.

2.2.2. Selection Nearness Principle. The principle of maximum membership and selection nearness principle are often used to recognize fuzzy diagnosis. In this paper, we use the selection nearness principle to detect the faults.

Let $A_{i}$ (standard fuzzy pattern) and $B$ (to be identified fuzzy object) be the fuzzy subsets, in which $i=1,2, \ldots, n$. If $i_{0}$ is present to make (8) establishment, then $B$ and $A_{i_{0}}$ are considered closest to each other, and $B$ is judged as $A_{i_{0}}$ class. Consider

$$
N\left(A_{i_{0}}, B\right)=\max \left\{N\left(A_{1}, B\right), N\left(A_{2}, B\right), \ldots, N\left(A_{n}, B\right)\right\} .
$$

In this paper, the Hamming approach degrees are used to calculate $N(A, B)$ :

$$
N(A, B)=1-\frac{1}{n} \sum_{k=1}^{n}\left|A\left(x_{k}\right)-B\left(x_{k}\right)\right|
$$

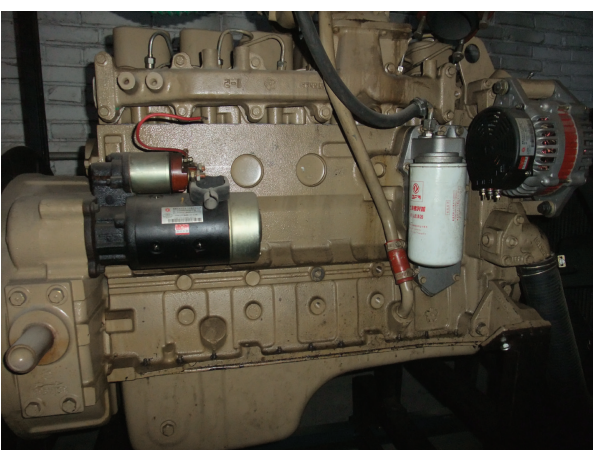

FIgURE 4: The experimental engine.

The greater the approach degrees $N(A, B)$ are, the more similar the two fuzzy subsets are and vice versa worse. In fuzzy diagnosis, each cluster center of known fault samples is firstly obtained by using FCM algorithm, and then the approach degrees between tested samples and cluster center are calculated. Finally, the class of tested samples is determined.

\section{Experimental Validations}

In this paper, we used the Cummins 6BT engine as experimental subjects, and there is a rotation speed sensor in its flywheel housing which can capture the speed signal. The experimental engine is shown in Figure 4. In this engine, the fourth channel bearing of crankshaft was set as failure bearing. In order to simulate a variety of wear conditions, four kinds of bearings with the gap are set to normal ( 0.08 $0.1 \mathrm{~mm})$, slight $(0.15-0.2 \mathrm{~mm})$, moderate $(0.22-0.26 \mathrm{~mm})$, and severe $(0.4-0.5 \mathrm{~mm})$. The acceleration sensor was placed on the left side of the fourth channel main bearing at the junction of the sump and cylinder. The vibration signal during engine acceleration process was collected. At the same time, the rotation speed of the crankshaft was calculated by speed sensor in the flywheel housing, and the sampling frequency was $20000 \mathrm{~Hz}$.

After we collected the signals of rotation speed and vibration, we used the method of speed signal processing to eliminate the error from the missing or additional pulse and then resampled the vibration signal by cubic spline interpolation method. The vibration signal in time domain of uniform acceleration process can be obtained. According to this process, five groups of data were collected in each kind of wear. The first three groups of signals were used as known fault samples, and the rest would be classified as fault sample data. Then, the vibration signals were analyzed using order tracking in the whole work cycle. It shows the order tracking spectrum of the first group of known samples with different conditions in Figure 5. As apparent from this figure, in the four kinds of conditions, the energy of vibration signal is mainly concentrated when the rotation speed is $2000 \mathrm{rpm}$. In the order band of $(0-150)$, with the deepening of the fault, 


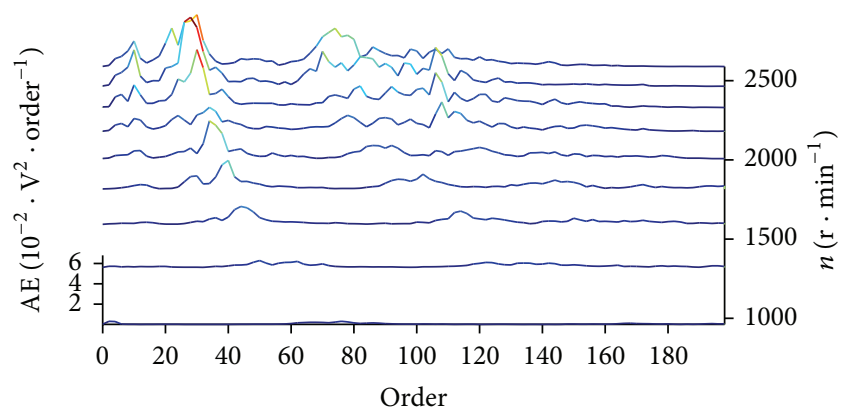

(a)

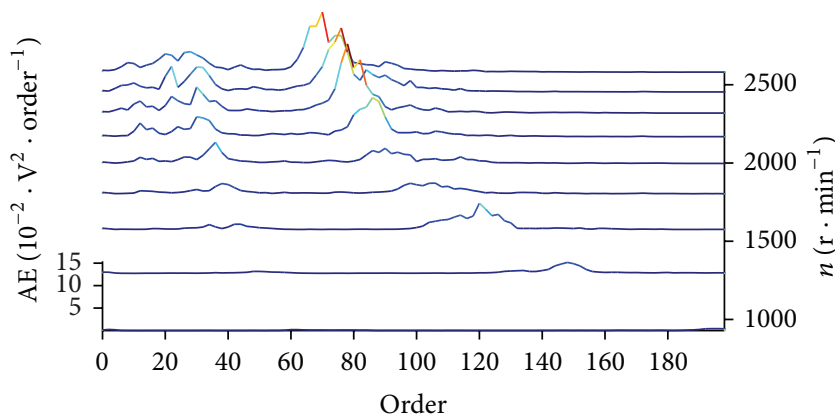

(c)

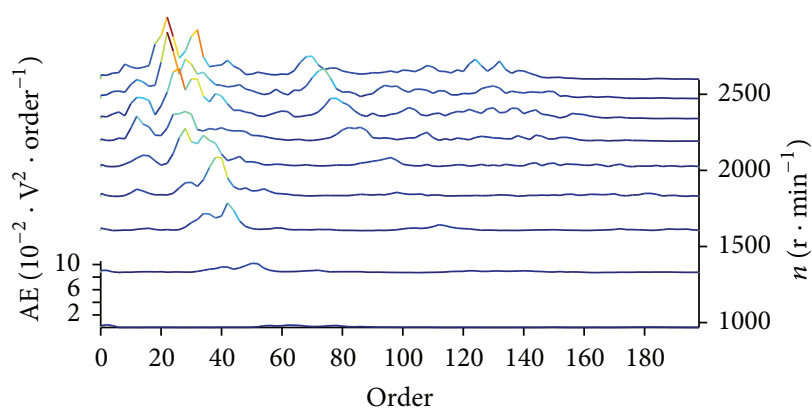

(b)

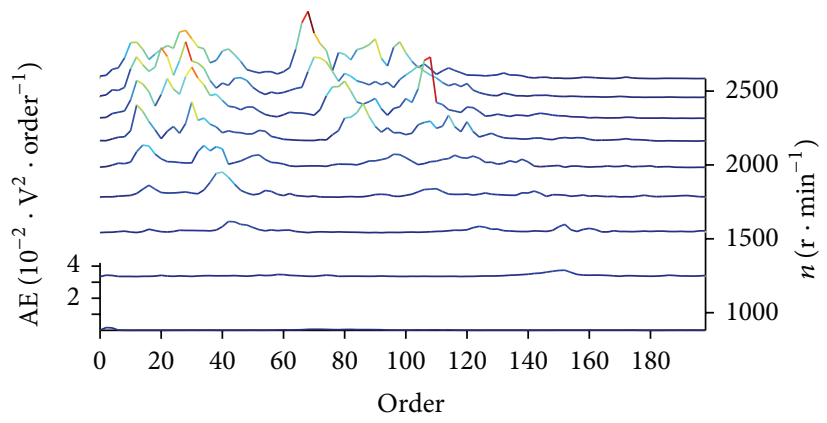

(d)

FIGURE 5: Order tracking spectrum of different bearing clearance (degree of wear: (a) normal, (b) slight, (c) moderate, and (d) severe).

there is a variation rule in the energy distribution of different order band. So, the accumulated energy in the order bands of (0-150), (0-20), (20-60), (60-100), (100-120), and (120-150) was used as the features, and the feature vector of the order band of the first 3 groups' known fault sample data is shown as follows in a matrix $X$ :

$$
X=\left[\begin{array}{llllll}
4.0165 & 0.3680 & 0.7626 & 2.1978 & 0.6465 & 0.3360 \\
4.1761 & 0.3221 & 0.5268 & 2.2695 & 0.8346 & 0.5048 \\
4.2169 & 0.3565 & 0.5548 & 2.3047 & 0.8693 & 0.4677 \\
4.5499 & 0.6053 & 1.0953 & 2.3349 & 0.3323 & 0.5561 \\
4.4224 & 0.5101 & 1.2202 & 2.4301 & 0.3574 & 0.4023 \\
5.0879 & 0.8516 & 1.0642 & 2.5907 & 0.3732 & 0.6409 \\
5.7589 & 0.6778 & 0.6708 & 4.1652 & 0.4309 & 0.1310 \\
4.6537 & 0.4090 & 0.4355 & 3.4701 & 0.4693 & 0.1227 \\
6.0114 & 0.7069 & 0.7270 & 4.3015 & 0.4341 & 0.1816 \\
3.2437 & 0.5350 & 0.5040 & 1.7425 & 0.5213 & 0.2086 \\
3.8354 & 0.7041 & 0.7217 & 2.1128 & 0.5153 & 0.1775 \\
3.9487 & 0.7034 & 0.5767 & 2.2935 & 0.5064 & 0.1719
\end{array}\right] .
$$

After these, we used FCM to classify the samples. Firstly, the feature vector matrix $X$ would be normalized to obtain the initial membership matrix. We used the following formula to normalize it:

$$
x_{i j}=\frac{x_{i j}-\min \left(x_{j}\right)}{\max \left(x_{j}\right)-\min \left(x_{j}\right)},
$$

where $x_{j}$ is the $j$ th column's data of the feature matrix $X$. The denominator value is the difference between the maximum value and the minimum value of the $j$ th column in the original matrix data. The data is converted into $[0,1]$ closed interval by a standardized treatment. Then, the initial membership matrix $\widehat{X}$ can be obtained by (13).

Hence, $\widehat{X}$ was used as the input of FCM clustering. In practice, the appropriate $m$ has noise suppression and smoothing effect of membership function. Usually, the range of $m$ is $[1.1,5]$. It is difficult to decide the values of $m$. Most researchers have recommended $m=2$ based on empirical studies [13]. A physical explanation of the FCM algorithm with $m=2$ was given in [14]. So, we choose $m=2$ in this paper. Because the set of fault classes was \{normal, slight, moderate, severe\}, the number of clusters was set as 4 . The stop condition of iteration was $\varepsilon=10^{-5}$. The feature parameter matrix was calculated by using FCM. After 14 iterations, the value of the objective function was 0.8179 , and the known fault samples membership matrix $U$ was obtained as follows: 
TABLE 1: Diagnosis results of sample under test.

\begin{tabular}{|c|c|c|c|c|c|c|c|c|c|c|c|}
\hline \multirow{2}{*}{ Number } & \multicolumn{6}{|c|}{ Features of the sample data } & \multicolumn{4}{|c|}{ Approach degree } & \multirow{2}{*}{ Classification results } \\
\hline & & & & & & & Normal & Slight & Moderate & Severe & \\
\hline 1 & 4.0400 & 0.4239 & 0.7414 & 2.1345 & 0.6874 & 0.4030 & 0.81 & 0.55 & 0.62 & 0.56 & Yes \\
\hline 2 & 4.3626 & 0.3592 & 0.8570 & 2.2606 & 0.7641 & 0.4970 & 0.90 & 0.60 & 0.66 & 0.59 & Yes \\
\hline 3 & 4.5474 & 0.6386 & 1.1611 & 2.4145 & 0.3372 & 0.4678 & 0.62 & 0.90 & 0.55 & 0.51 & Yes \\
\hline 4 & 4.5546 & 0.6696 & 1.0654 & 2.5060 & 0.3047 & 0.4386 & 0.63 & 0.93 & 0.55 & 0.52 & Yes \\
\hline 5 & 5.9667 & 0.6100 & 0.8035 & 4.4526 & 0.3446 & 0.1232 & 0.69 & 0.66 & 0.89 & 0.43 & Yes \\
\hline 6 & 6.7985 & 0.7553 & 0.7659 & 5.0582 & 0.4599 & 0.1558 & 0.55 & 0.62 & 0.83 & 0.33 & Yes \\
\hline 7 & 2.9669 & 0.4705 & 0.4396 & 1.7863 & 0.3588 & 0.1473 & 0.67 & 0.65 & 0.42 & 0.89 & Yes \\
\hline 8 & 3.1679 & 0.5943 & 0.5363 & 1.6174 & 0.4891 & 0.2050 & 0.61 & 0.49 & 0.36 & 0.87 & Yes \\
\hline
\end{tabular}

TABLE 2: Diagnosis results in nonentire work cycle mode.

\begin{tabular}{|c|c|c|c|c|c|c|c|c|c|c|c|}
\hline \multirow{2}{*}{ Number } & \multicolumn{6}{|c|}{ Features of the sample data } & \multicolumn{4}{|c|}{ Approach degree } & \multirow{2}{*}{ Classification results } \\
\hline & & & & & & & Normal & Slight & Moderate & Severe & \\
\hline 1 & 5.4550 & 0.7025 & 0.9752 & 2.4580 & 0.7988 & 0.5205 & 0.78 & 0.65 & 0.61 & 0.58 & Yes \\
\hline 2 & 6.0856 & 0.6027 & 1.2101 & 2.7543 & 0.9132 & 0.6054 & 0.85 & 0.59 & 0.64 & 0.65 & Yes \\
\hline 3 & 6.3027 & 0.7899 & 1.4250 & 3.0012 & 0.4125 & 0.6741 & 0.66 & 0.90 & 0.63 & 0.51 & Yes \\
\hline 4 & 5.6725 & 0.8221 & 1.1558 & 2.6998 & 0.3985 & 0.5962 & 0.56 & 0.86 & 0.60 & 0.65 & Yes \\
\hline 5 & 7.6145 & 0.8500 & 0.9948 & 4.9538 & 0.5110 & 0.3048 & 0.65 & 0.70 & 0.76 & 0.66 & Yes \\
\hline 6 & 8.0366 & 0.7993 & 0.8766 & 5.6023 & 0.4599 & 0.2985 & 0.58 & 0.65 & 0.60 & 0.40 & No \\
\hline 7 & 4.1172 & 0.5905 & 0.6987 & 2.2458 & 0.3142 & 0.2679 & 0.61 & 0.45 & 0.52 & 0.83 & Yes \\
\hline 8 & 4.1931 & 0.6213 & 0.8052 & 1.9871 & 0.4585 & 0.3210 & 0.67 & 0.58 & 0.45 & 0.81 & Yes \\
\hline
\end{tabular}

$$
U=\left[\begin{array}{llllllllllll}
0.2628 & 0.0124 & 0.0106 & 0.0092 & 0.1067 & 0.1122 & 0.0028 & 0.3387 & 0.0123 & 0.7654 & 0.9103 & 0.9396 \\
0.1256 & 0.0080 & 0.0063 & 0.9780 & 0.7552 & 0.7029 & 0.0020 & 0.1211 & 0.0115 & 0.0669 & 0.0345 & 0.0196 \\
0.5381 & 0.9736 & 0.9780 & 0.0064 & 0.0685 & 0.0792 & 0.0015 & 0.1891 & 0.0074 & 0.1169 & 0.0282 & 0.0197 \\
0.0735 & 0.0060 & 0.0051 & 0.0064 & 0.0696 & 0.1057 & 0.9938 & 0.3511 & 0.9688 & 0.0508 & 0.0270 & 0.0211
\end{array}\right]
$$

The cluster center $C$ was calculated as follows:

$$
C=\left[\begin{array}{llllll}
0.1969 & 0.6031 & 0.2246 & 0.1626 & 0.3377 & 0.1261 \\
0.5035 & 0.5905 & 0.8641 & 0.2699 & 0.0386 & 0.7821 \\
0.3375 & 0.0477 & 0.1720 & 0.2149 & 0.8988 & 0.6499 \\
0.9207 & 0.6657 & 0.3188 & 0.9462 & 0.1910 & 0.0670
\end{array}\right]
$$

The cluster center could be available as standard crankshaft bearing fault modes. In $C$, the first, second, third, and fourth lines are the normal, slight, moderate, and severe wear cluster center of sample data, respectively. Another two groups of samples were used as diagnosed data $B$ after being normalized by (13). According to (11), Hamming approach degrees between $B$ and the standard mode $C$ were calculated and used to decide the class of test samples. Parts of the results were shown in Table 1 by this method. Bold part of the table was the maximum approach degree of sample data.

As a comparison, we analyzed the vibration signal from $1000 \mathrm{rpm}$ to $2500 \mathrm{rpm}$ using nonentire work cycle mode method. The order tracking spectrum is computed and the accumulated energy in the order bands of (0-150), (0-20), (20-60), (60-100), (100-120), and (120-150) was also used as the features. Parts of the results were shown in Table 2 by this method. Bold words in Table 2 were the maximum approach degree of sample data.

Through statistics in 200 groups of unknown samples, the classification accuracy was $89 \%$ in our method but that was $69 \%$ in nonentire work cycle mode. This reveals that our method has more accuracy classification.

\section{Conclusions}

In this paper, a method is introduced combining order tacking and FCM, in which the order tracking spectrum of the entire work cycle follows with the characteristics of reciprocating engine; hence, order tracking spectrum can well characterize the vibration signal of the engine during acceleration process, and the accumulated energy of different order band can reflect the features of diesel engine faults. Based on the results of order tacking, the FCM clustering algorithm can 
generate diagnostic criteria from existing troubleshooting cases; then, the method by Hamming approach degrees to pattern recognition is simple and effective in fault detection. The experiments show that the method can work well. A comparison with the method of nonentire work cycle shows our method has more accuracy classification.

\section{Conflict of Interests}

The authors declare that there is no conflict of interests regarding the publication of this paper.

\section{Acknowledgment}

This work was funded by the National Natural Science Foundation of China (no. 60674111). This support is gratefully acknowledged.

\section{References}

[1] L.-L. Kong, Y.-K. Xiao, G.-P. Jiang, B.-M. Wang, L.-L. Zhang, and T. Xia, "Analysis and comparison of engine steady and unsteady vibration signal," Transactions of Chinese Society for Internal Combustion Engines, vol. 24, no. 1, pp. 72-77, 2006.

[2] W. Yang, C. Little, and R. Court, "S-transform and its contribution to wind turbine condition monitoring," Renewable Energy, vol. 62, pp. 137-146, 2014.

[3] K. Aharamuthu and E. P. Ayyasamy, "Application of discrete wavelet transform and Zhao-Atlas-Marks transforms in non stationary gear fault diagnosis," Journal of Mechanical Science and Technology, vol. 27, no. 3, pp. 641-647, 2013.

[4] L. Lin and F. Chu, "HHT-based AE characteristics of natural fatigue cracks in rotating shafts," Mechanical Systems and Signal Processing, vol. 26, no. 1, pp. 181-189, 2012.

[5] J. Antoni, J. Daniere, and F. Guillet, "Effective vibration analysis of IC engines using cyclostationarity. Part I-a methodology for condition monitoring," Journal of Sound and Vibration, vol. 257, no. 5, pp. 815-837, 2002.

[6] J. Wang, R. X. Gao, and R. Yan, "Multi-scale enveloping order spectrogram for rotating machine health diagnosis," Mechanical Systems and Signal Processing, vol. 46, no. 1, pp. 28-44, 2014.

[7] P. Borghesani, P. Pennacchi, S. Chatterton, and R. Ricci, "The velocity synchronous discrete Fourier transform for order tracking in the field of rotating machinery," Mechanical Systems and Signal Processing, vol. 44, no. 1-2, pp. 118-133, 2014.

[8] P. Borghesani, P. Pennacchi, R. B. Randall, and R. Ricci, “Order tracking for discrete-random separation in variable speed conditions," Mechanical Systems and Signal Processing, vol. 30, pp. 1-22, 2012.

[9] H. Kang, J. Luan, Y. Tian, H. Zheng, and J. Cao, "Application of the order tracking analysis in gear wearing," Journal of Vibration and Shock, vol. 25, no. 4, pp. 112-118, 2006.

[10] H. K. Alaei and K. Salahshoor, "A new integrated on-line fuzzy clustering and segmentation methodology with adaptive PCA approach for process monitoring and fault detection and diagnosis," Soft Computing, vol. 17, no. 3, pp. 345-362, 2013.

[11] S. Bahrampour, B. Moshiri, and K. Salahshoor, "Weighted and constrained possibilistic C-means clustering for online fault detection and isolation," Applied Intelligence, vol. 35, no. 2, pp. 269-284, 2011.
[12] H. Zhang and J. Pu, "Robust image segmentation using KFCM with noise restrained," in Proceedings of the 6th World Congress on Intelligent Control and Automation, pp. 10210-10214, June 2006.

[13] J. Yu, Q. Cheng, and H. Huang, "Analysis of the weighting exponent in the FCM," IEEE Transactions on Systems, Man, and Cybernetics, Part B: Cybernetics, vol. 34, no. 1, pp. 634-639, 2004.

[14] J. C. Bezdek, "A physical interpretation of fuzzy ISODATA," IEEE Transactions on Systems, Man, and Cybernetics, vol. 6, no. 5, pp. 387-390, 1976. 

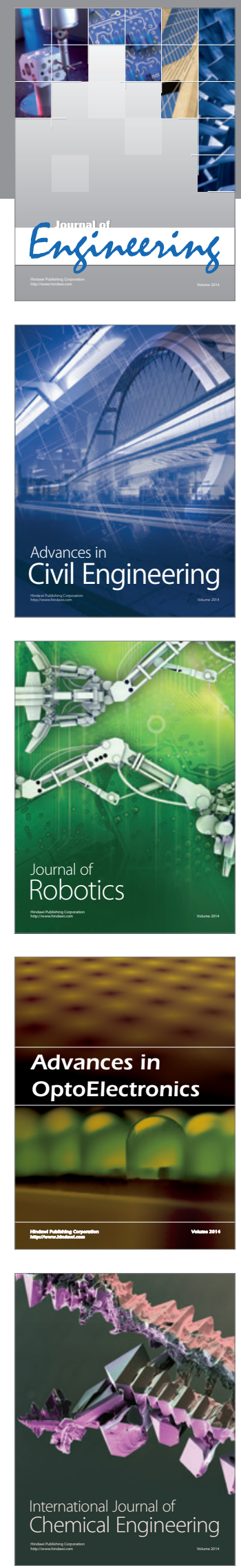

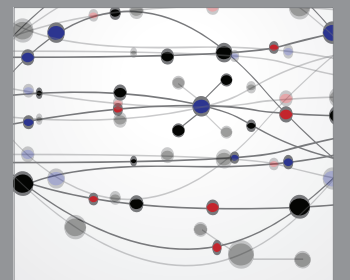

The Scientific World Journal
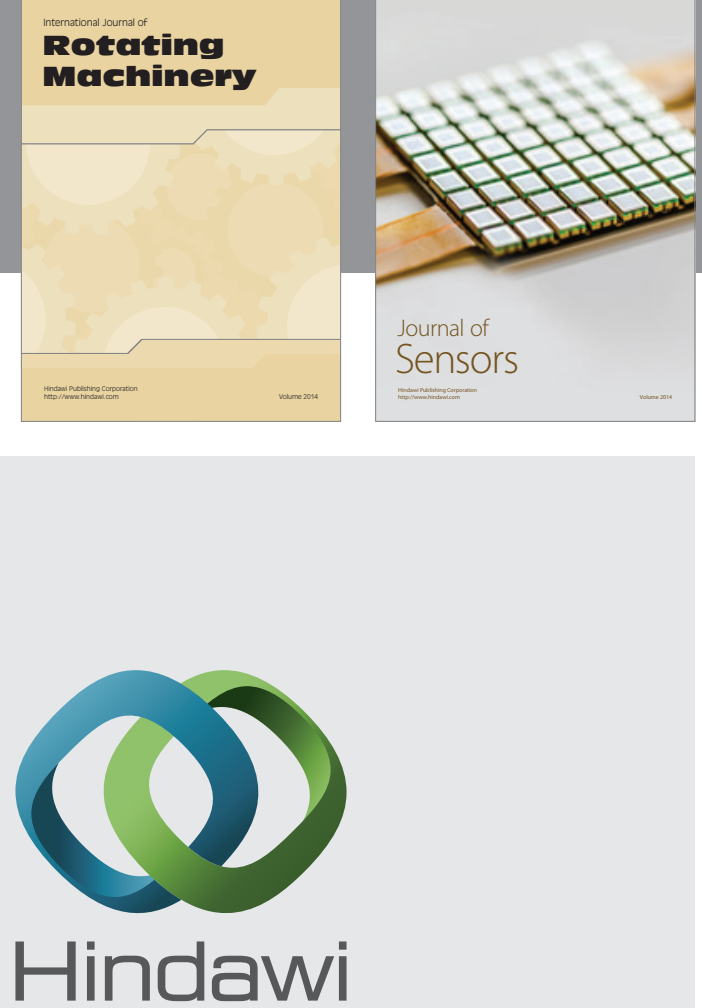

Submit your manuscripts at http://www.hindawi.com
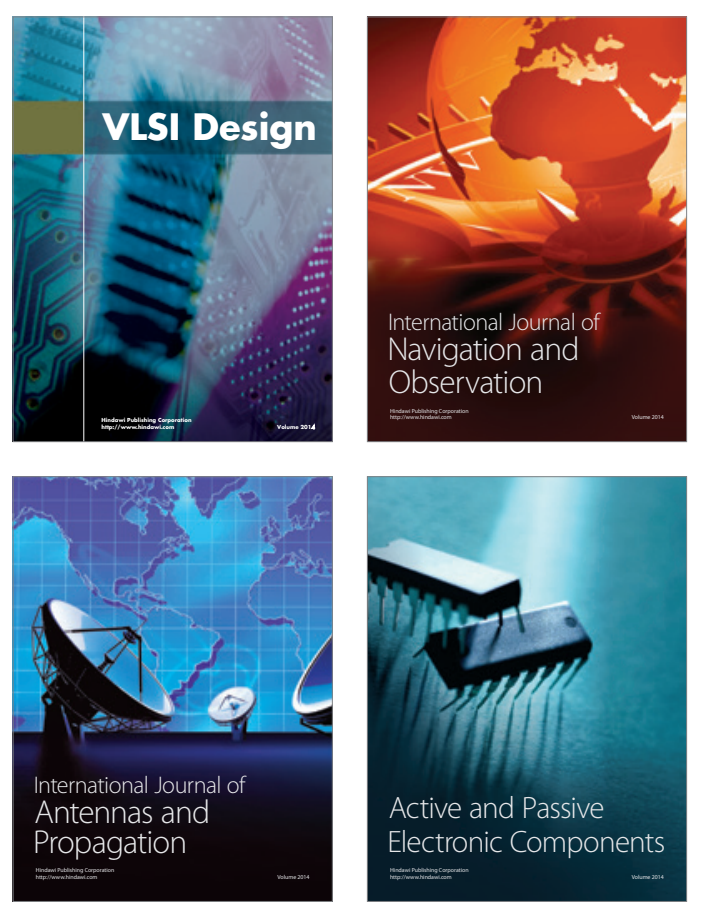
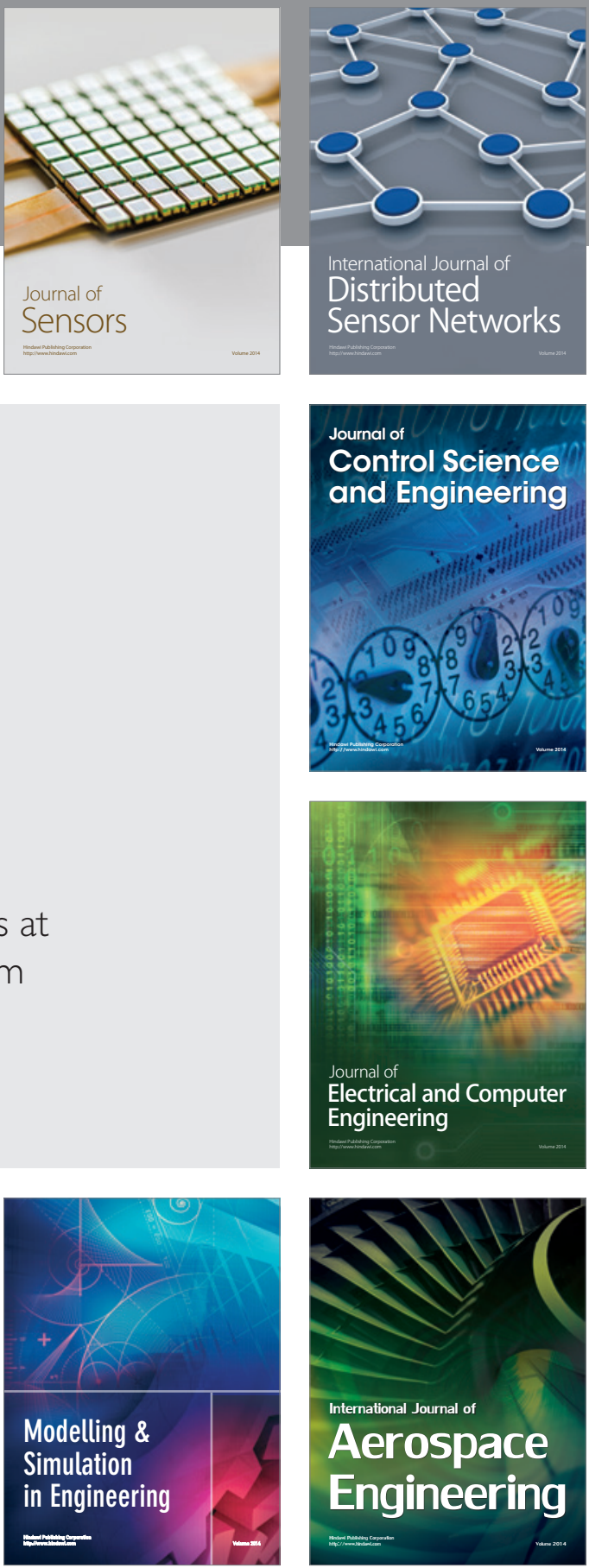

Journal of

Control Science

and Engineering
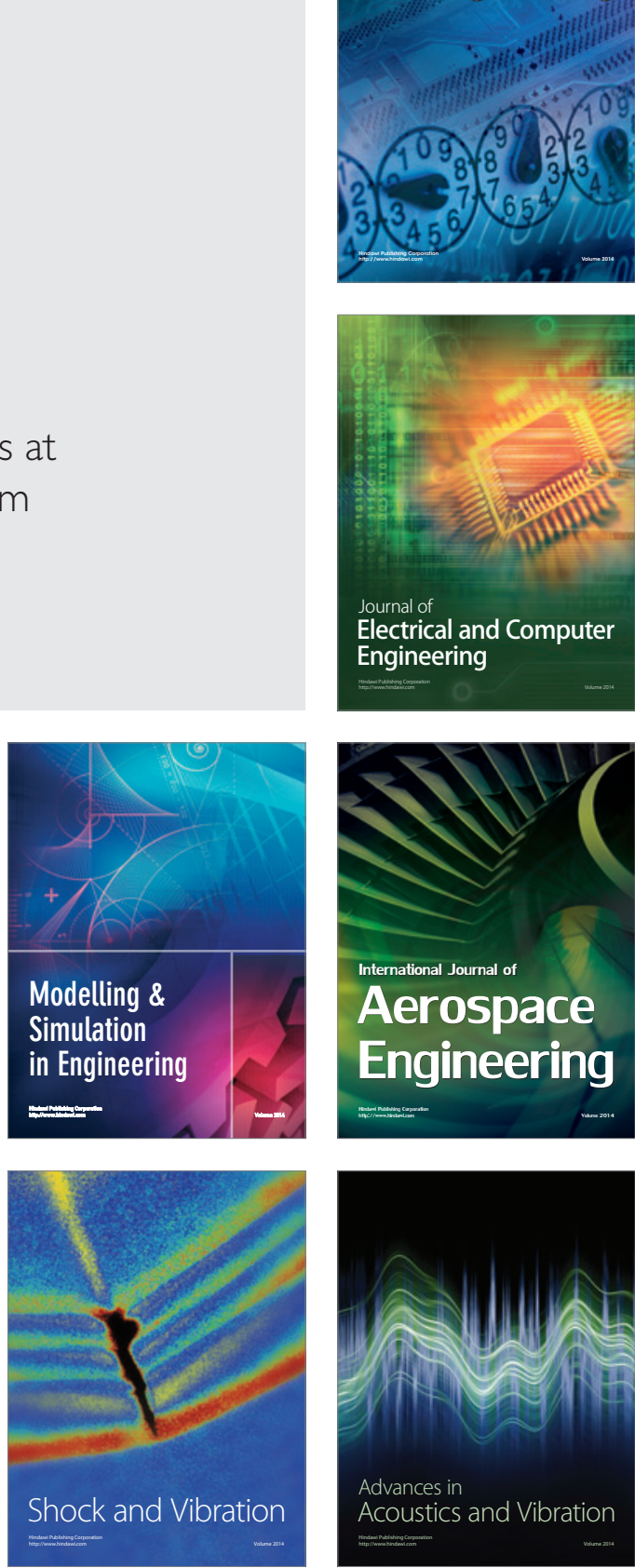